\title{
Bilateral thoracoscopic cervical sympathectomy for the treatment of recurrent polymorphic ventricular tachycardia
}

\section{A J Turley, J Thambyrajah, A A Harcombe}

Heart 2005;91:15-17. doi: 10.1136/hrt.2004.039271

Congenital long QT syndrome (LQTS) is a disorder of prolonged cardiac repolarisation, manifest by a prolonged QT interval and characterised by recurrent presyncope/ syncope, polymorphic ventricular tachycardia (PMVT), or sudden cardiac death. A 46 year old woman with no family history of sudden death or deafness presented with recurrent syncope. Physical examination and electrolytes were normal and a 12 lead ECG showed a corrected QT interval of $458 \mathrm{~ms}$. A monitored syncopal episode documented PMVT. Despite potassium and magnesium supplements, $\beta$ blockade, implantation of a single then dual chamber implantable cardioverter defibrillator (ICD), amiodarone, nicorandil, and mexiletine, the patient continued to experience arrhythmia storms, receiving more than 700 ICD discharges over seven months. She was ultimately treated successfully with bilateral thoracoscopic cervicothoracic sympathectomies. This is the first reported bilateral thoracoscopic treatment of a patient with LQTS and symptomatic life threatening ventricular tachyarrhythmias refractory to current pharmacological and pacing techniques.

C ongenital long QT syndrome (LQTS) is a disorder of prolonged cardiac repolarisation caused by a heterogeneous group of cardiac potassium and sodium ion channel mutations. It is characterised by recurrent presyncope/syncope, polymorphic ventricular tachycardia (PMVT)/ torsade de pointes, or sudden cardiac death and is manifested by a prolonged QT interval. We present a case of LQTS refractory to conventional pharmacological and pacing techniques.

\section{CASE REPORT}

A 46 year old woman was admitted with a six hour history of recurrent syncope. There was a history of syncope 12 years previously with recurrent episodes of presyncope and palpitations in the intervening years. She had hyperlipidaemia but no other atherosclerotic risk factors and no family history of sudden death or congenital deafness. Chlorphenamine was her only drug treatment. Physical examination and electrolytes were normal and a 12 lead ECG (fig 1) showed normal mean frontal QRS axis, inferolateral ST/T abnormalities, and corrected QT interval $458 \mathrm{~ms}$. A monitored syncopal episode documented PMVT, treated with intravenous magnesium and amiodarone. Echocardiography showed normal cardiac structure and function. Angiography showed normal coronary arteries but was complicated by traumatic dissection of the right coronary artery requiring immediate percutaneous intervention. After the patient was appropriately counselled a single lead implantable cardioverter defibrillator (ICD) (Ventak Prizm 2 VR, Guidant) was inserted and amiodarone was discontinued. An exercise test excluded exercise related repolarisation abnormalities and the patient was prescribed nadolol and discharged.

Over the next few months, the patient had numerous readmissions with symptomatic PMVT requiring ICD discharge (figs 2 and 3). Multiple PMVT storms occurred, despite adjustments to the pacing rate, correction of unexplained hypokalaemia with amiloride, and the addition of nicorandil and mexiletine. Mexiletine was subsequently discontinued. Apparent success of a percutaneous temporary left stellate ganglion block at the C7-T1 level prompted a definitive computed tomography guided radiofrequency approach, resulting in a two week period before the next storm. The ICD was upgraded to a dual chamber Vitality DR unit (Guidant) pacing at 100 beats/min with a long detection time for ventricular tachycardia (15 seconds). Nadolol was replaced with metoprolol.

There followed a quiescent period lasting three weeks but then, almost three months after she first presented, the patient was readmitted with another PMVT storm. Further amiodarone failed to prevent daily ICD discharges; thus, it was discontinued and the dose of metoprolol was increased. The patient then underwent left thoracoscopic cardiac sympathectomy at Tl-T4, producing a mild Horner's syndrome, but this did not reduce the frequency of PMVT. At this point her ICD generator reached its "end of life" and was replaced. Diagnostic angiography excluded in-stent restenosis. Before transplantation was considered, the patient underwent right thoracoscopic cardiac sympathectomy from the lower half of the stellate ganglion to T4. This was associated with a dramatic improvement in her symptoms and she was discharged shortly afterwards.

During the seven month period when the patient was effectively living on the coronary care unit, she became anxious and depressed, with suicidal ideation, requiring psychiatric support, antidepressants, and benzodiazepines. Her husband was also severely affected, losing his employment as a direct result. At present, the patient has not been readmitted in over 11 months. She is regularly monitored in the outpatient clinic and reports considerable improvements in both physical and psychological states, with plans to return to work soon.

\section{DISCUSSION}

Patients with certain forms of congenital LQTS are at high risk of sudden death. Immediate management includes withdrawal of precipitating agents and correction of electrolyte abnormalities. Subsequent treatment aims at preventing life threatening ventricular tachyarrhythmias. The role of $\beta$ blockers as first line agents has been supported by several studies. ${ }^{12}$ Adjunctive treatment with an atrial based permanent pacemaker at a rate to normalise the QT interval is indicated for patients who remain symptomatic or where

Abbreviations: ICD, implantable cardioverter defibrillator; LQTS, long QT syndrome; PMVT, polymorphic ventricular tachycardia 


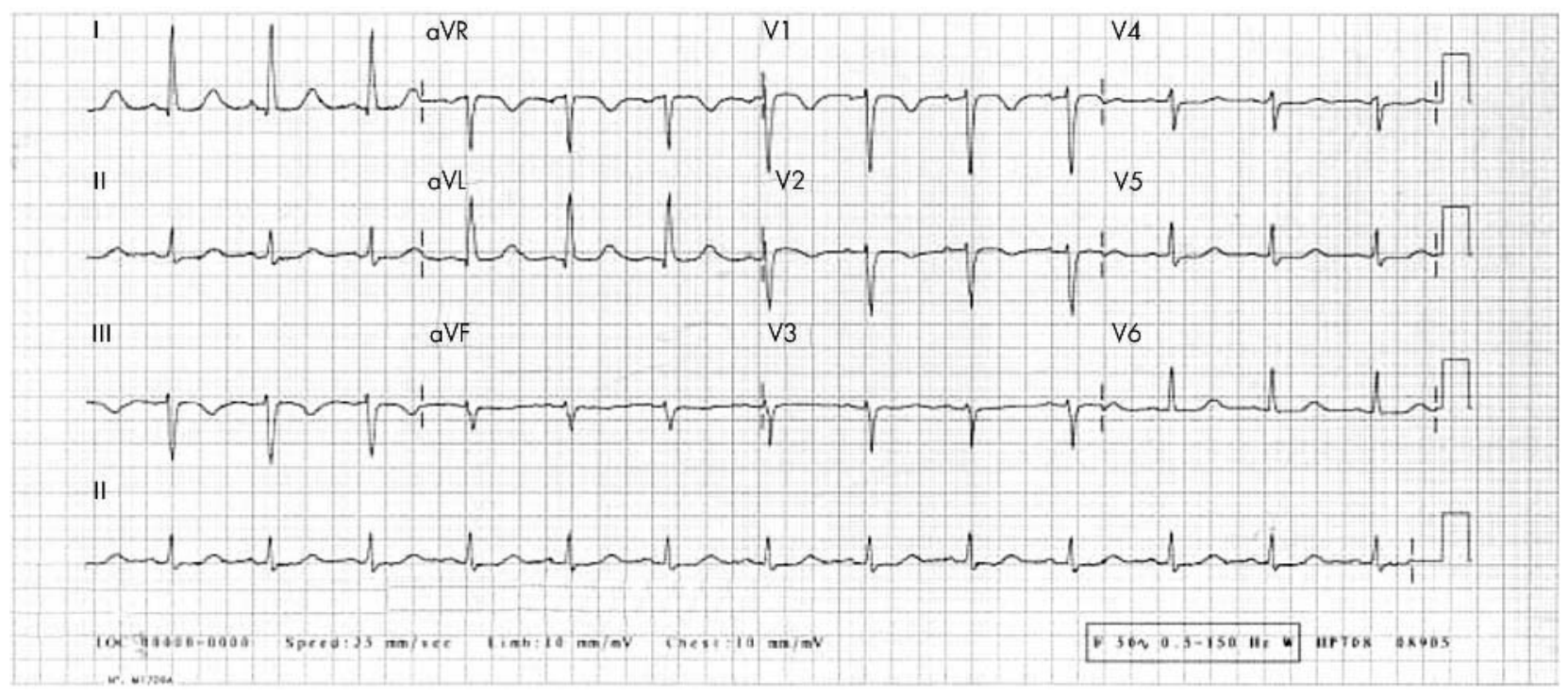

Figure 1 Twelve lead ECG showing prolonged corrected QT interval.

bradycardia is a precipitant of PMVT. ${ }^{3}$ The increasing availability of dual chamber ICD technology with antitachycardia pacing facilities coupled with the potentially fatal outcome of the failure of pharmacological treatment has led to the use of these devices in many symptomatic patients. ${ }^{45}$

A growing understanding of the aetiology of subgroups of congenital LQTS has stimulated the development of treatments specifically targeted at mutant ion channels. LQT3 relates to an increase in sodium current, whereas LQTI and LQT2 arise from deficient potassium currents-hence, the use of drugs that activate potassium currents or selectively block excess sodium currents. Nicorandil increases the outward potassium current of myocardial cells thereby reducing early afterdepolarisations, a mechanism implicated in the development of PMVT. Nicorandil has been effective in this clinical setting. ${ }^{6}$ Similar anecdotal evidence applies to sodium channel blockers such as mexiletine. ${ }^{7}$ However, there are no data from randomised controlled trails and our patient did not improve with either of these medications. The management of this case was further complicated as the patient did not appear to have a clearly defined form of LQTS. Although a dual chamber ICD might have been indicated as the first implant, an upgrade to this modality did not reduce the frequency of PMVT. Ultimately the patient received more than 700 discharges from two devices.

Autonomic imbalance has been implicated in the pathogenesis of LQTS. ${ }^{8}$ Animal studies indicate that the sympathetic nervous system, mediated largely through the "qualitatively" dominant left stellate ganglion, has a greater adrenergic influence on the ventricles than the right. ${ }^{9}$ High left cervical sympathetic denervation is a highly effective antiadrenergic surgical method, achieving adequate cardiac denervation by the removal of the first four to five left thoracic ganglia and the lower half of the left stellate

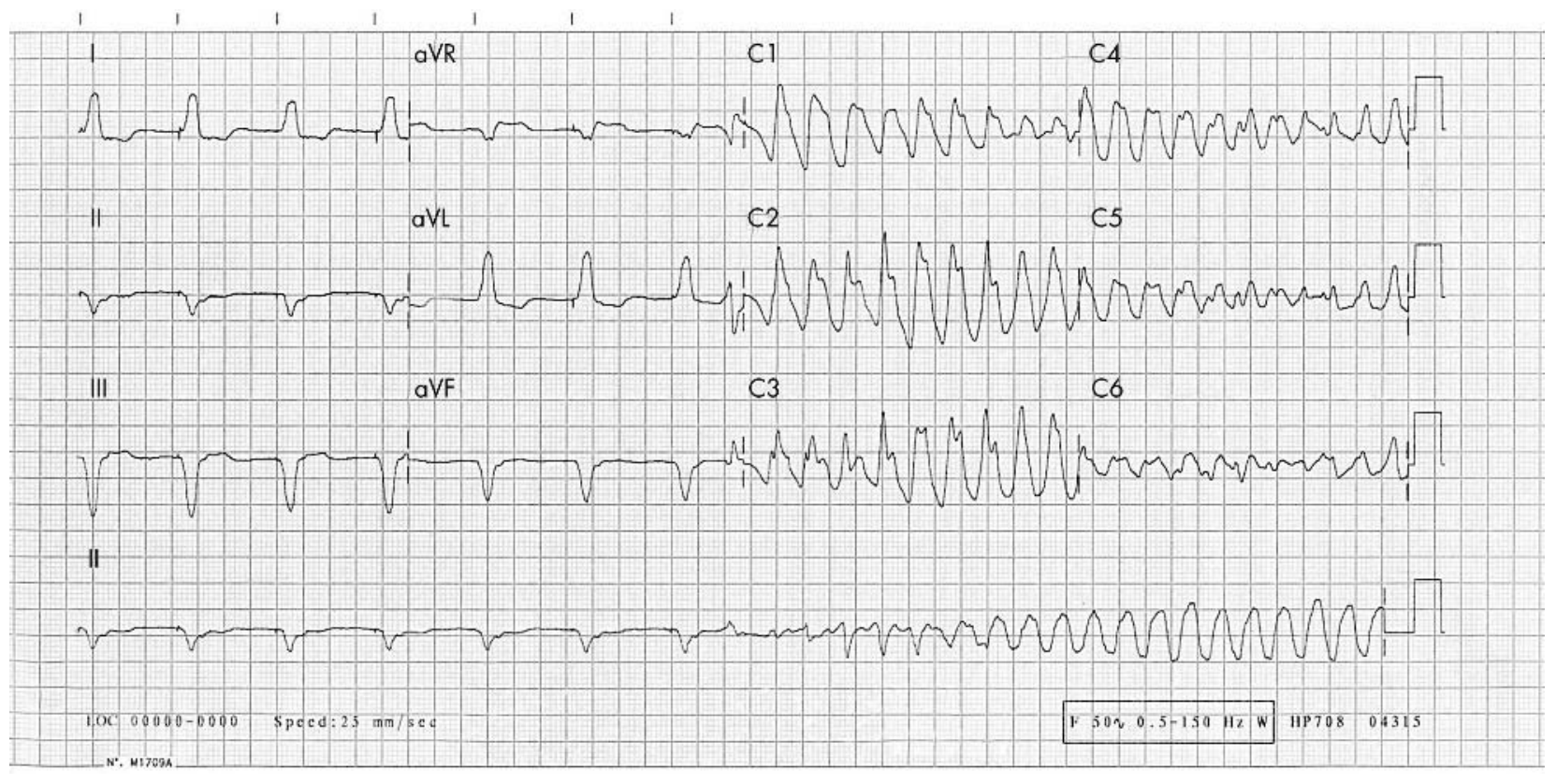

Figure 2 Onset of polymorphic ventricular tachycardia during 12 lead ECG recording at time of ventricular based device discharge. 

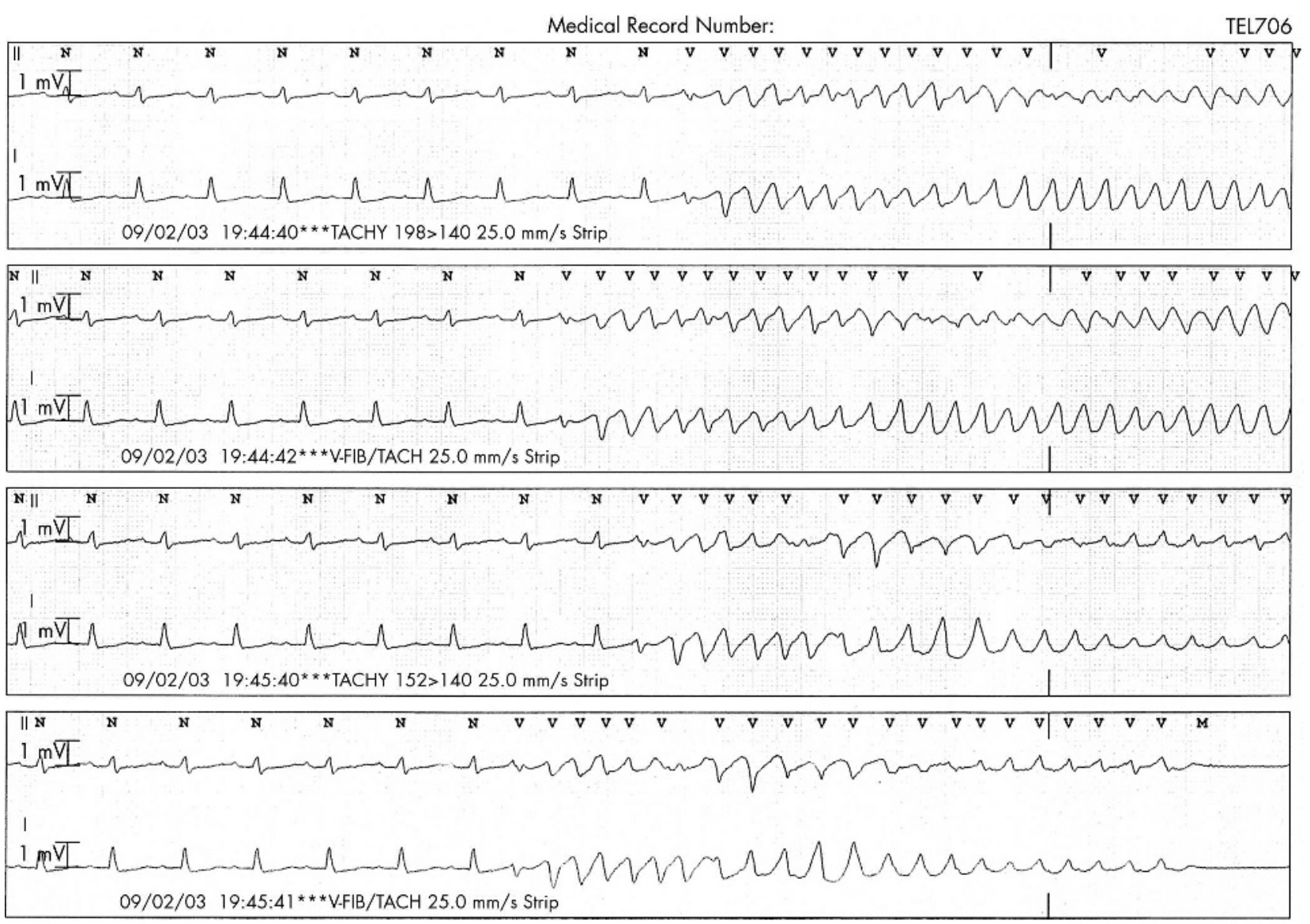

Figure 3 Frequent spontaneous episodes of polymorphic ventricular tachycardia during telemetry monitoring in coronary care unit.

ganglion. Left cervical sympathetic denervation does not affect the QT interval and its mechanism of action may involve a reduction in afterdepolarisations. ${ }^{8}$ This surgical approach was associated with a significant decrease in adverse cardiac events and a $94 \%$ five year survival in a case series of 85 patients with LQTS. ${ }^{8}$ However, it did not abolish events in our patient and a right thoracoscopic cervicothoracic sympathectomy was also performed.

Although bilateral open cervicothoracic sympathetic ganglionectomy has previously been reported, ${ }^{10}$ this is the first reported case of successful bilateral thoracoscopic cervicothoracic sympathectomies in the treatment of a patient with LQTS and symptomatic life threatening ventricular tachyarrhythmias refractory to current pharmacological and pacing techniques.

\section{ACKNOWLEDGEMENTS}

We are indebted to Mr Alec Clason for performing both thoracoscopic cervicothoracic sympathectomies, to Dr Simon Eldabe for performing local anaesthesia and computed tomography guided procedures, and to Professor Peter Schwartz for his invaluable advice and support.

\section{Authors' affiliations}

A J Turley, J Thambyrajah, A A Harcombe, Cardiothoracic Division, The James Cook University Hospital, Middlesbrough, UK

Correspondence to: Dr Andrew J Turley, Cardiothoracic Division, The James Cook University Hospital, Marton Road, Middlesbrough TS4 3BW, UK; andrew.turley@stees.nhs.uk
Accepted 15 July 2004

\section{REFERENCES}

1 Dorostkar PC, Eldar M, Belhassen B, et al. Long-term follow-up of patients with long-QT syndrome treated with beta-blockers and continuous pacing. Circulation 1999;100:2431-6.

2 Moss AJ, Schwartz PJ, Crampton RS, et al. The long QT syndrome: a prospective international study. Circulation 1985;71:17-21.

3 Moss AJ, Liu JE, Gottlieb S, et al. Efficacy of permanent pacing in the management of high-risk patients with long QT syndrome. Circulation 1991;84:1524-9.

4 Zareba W, Moss AJ, Daubert JP, et al. Implantable cardioverter defibrillator in high-risk long QT syndrome patients. J Cardiovasc Electrophysiol 2003; 14:337-41.

5 Schwartz PJ, Priori SG, Cerrone M, et al. Left cardiac sympathetic denervation in the management of high-risk patients affected by the long-QT syndrome. Circulation 2004; 109:1826-33.

6 Sato T, Hata Y, Yamamoto M, et al. Early afterdepolarization abolished by potassium channel opener in a patient with idiopathic long QT syndrome. J Cardiovasc Electrophysiol 1995;6:279-82.

7 Sicouri S, Antzelevitch D, Heilmann C, et al. Effects of sodium channel block with mexiletine to reverse action potential prolongation in in vitro models of the long term QT syndrome. J Cardiovasc Electrophysiol 1997;8: 1280-90.

8 Schwartz PJ, Locati EH, Moss AJ, et al. Left cardiac sympathetic denervation in the therapy of congenital long QT syndrome: a worldwide report. Circulation 1991;84:503-11.

9 Ben-David J, Zipes D. Differential response to right and left ansae subclaviae stimulation of early afterdepolarizations and ventricular tachycardia induced by cesium in dogs. Circulation 1988;78: 1241-50.

10 Lloyd R, Okada R, Stagg J, et al. The treatment of recurrent ventricular tachycardia with bilateral cervico-thoracic sympathetic-ganglionectomy: a report of two cases. Circulation 1974;50:382-8. 